\title{
An Experimental Investigation of the Effect of Slot Vortex on Plane Gate Vibration
}

\author{
Chunying Shen ${ }^{1,2, a}$, Shihua He ${ }^{*}$, , b , Wei Wang ${ }^{2, ~ c}$ and Yuelou Liu ${ }^{1, d}$ \\ ${ }^{1}$ Faculty of Electrical Power Engineering, Kunming University of Science and Technology, Kunming \\ 650500, China; \\ ${ }^{2}$ State Key Laboratory of Hydraulics and Mountain River Engineering, Sichuan University, Chengdu \\ 610065, China \\ ${ }^{*}$ Corresponding author \\ a86295700@qq.com, bhoxiwa@aliyun.com, profwangwei@sina.com, d1035896793@qq.com
}

\begin{abstract}
Keywords: vortex-induced vibration, plane gate, gate slot, submerged flow, 3D-PIV.
Abstract. An experimental investigation on vortex-induced vibration of plane gate with submerged flow is carried out by a combination of 3D-PIV technique and multi-channel vibrating data acquisition system. In order to explore the mechanism of gate vibration, the experiment is to synchronously test the distribution of fluid velocity field and vibration parameters of the plane gate with and without slot respectively. The experimental results show that the unstable vortex structure in the gate slot has certain incentive effects on the gate vibration and the flow fluctuation near the gate. The vibration displacement amplitude of the gate mainly depends on the central position and the energy of vortex located in the downstream of the gate.
\end{abstract}

\section{Introduction}

The plane gate is the most widely used in hydraulic engineering, but the sidewall is suddenly expanded due to the existence of the gate slot, which makes the water flow form the unstable vortex region inside the slot. The vortex inside the slot will affect the flow near the gate and further aggravate the vibration of the gate.

Research on the flow-induced vibration mechanism has made some achievements. Thang [1] indicated that the excitation mechanism of vertical-lift gate was attributed to the combined effect of shear-layer instabilities and motion-induced vortices shed at the leading edge of the gate bottom. Billeter [2] also confirmed this conclusion by experimental study. Kostecki [3] predicted the two-dimensional flow field in the vicinity of an underflow vertical lift gate using numerical combinative modeling of the vortex method and the boundary element method. Ozalp et al. [4]'s experiments in a large scale water channel were carried out in order to investigate the flow structure in rectangular, triangular and semi-circular cavity respectively using the PIV technique. In this paper, the mechanism of gate vibration and variation of hydraulic parameters with slot and without slot are studied by analyzing the vortices in the flow field behind plane gate.

Experimental Investigation is a main way to solve the problem of engineering hydraulics for complex flow behavior if the test equipment is suitable and precise. Highly accurate and three-dimensional particle image velocimetry (PIV) can help us to obtain the vortex information of transient velocity field by analyzing the flow image, which overcomes the deficiency of traditional single-point measuring apparatus. In recent years, the PIV technique finds wide application not only in the heat exchange field (e.g. Yataghene et al. [5]; Baum et al. [6]), but also in the field of water science(e.g.Tang et al. [7]; Druault et al.[8]; Ayati et al.[9]; Watanabe et al. [10]; Thien et al. [11]; Ferguson et al. [12]). Zhang et al. [13] obtained satisfactory results in researching the vortex distribution by PIV technique.

In this paper, the mechanism of fluid-induced vibration of a plane gate is investigated by synchronous combination testing of 3D-PIV technique and multi-channel vibrating data acquisition system to analyze the flow characteristics and corresponding gate vibration parameters, which has not been found in other studies. 


\section{Synchronous experiment setup}

The experimental study on vortex-induced vibration of a plane gate with submerged flow uses the three-dimensional particle imaging velocimetry system made by TSI Company, combining with the multi-channel vibrating data acquisition system. Two sets of instruments are applied to measure synchronously the real-time changes of flow field and the gate vibration parameters in order to investigate the reason of gate vibration amplitude, the impact of slot on gate vibration and the flow pattern.

3D-PIV is an advanced technology in testing the distribution of transient flow field. Because the test process does not interfere with the flow, it has the incomparable advantage over other traditional testing method, especially in the analysis of flow structure. INSIGHT 3G software and two special across-frame CCD cameras are used to shoot the flow field particle image which is processed according to the principle of autocorrelation and cross-correlation. Finally, the transient flow velocity vector diagram could be got through the post-processing software. In this paper, 3D-PIV is adopted to test the flow field behind a plane gate with submerged flow.

The multi-channel data acquisition instrument is used to acquire the gate vibration parameters. The acceleration sensors are laid on different positions of the upstream face of plane gate to reduce the impact of the downstream flow field. The testing point arrangement is shown in Fig. 1. The vibration acceleration, sampled interval 0.01s in the x-direction, is monitored and stored by DEWESOFT 7.1 software. Vibration velocity is obtained by integrating the acceleration variable, and the displacement obtained by integrating the velocity. The vibration spectrum of gate is got from the time history curve of displacement by FFT.
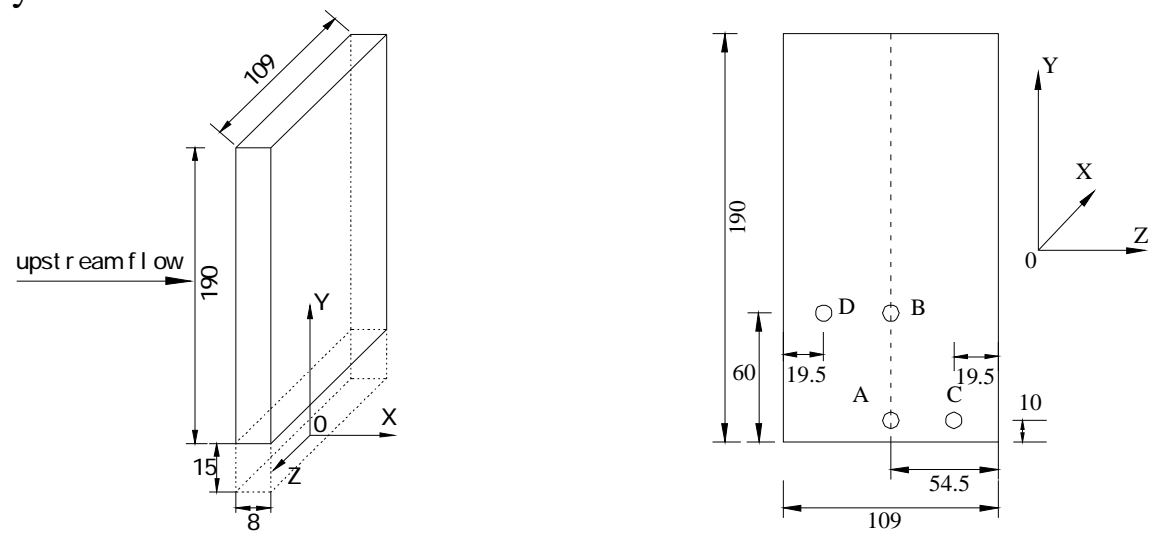

Fig.1. Coordinate system and vibration testing points arrangement (unit: $\mathrm{mm}$ )

The plane gate size is $109 \mathrm{~mm} \times 190 \mathrm{~mm} \times 8 \mathrm{~mm}$. For the test conditions without gate slots, the slots on both sides of the gate are filled with plasticine.

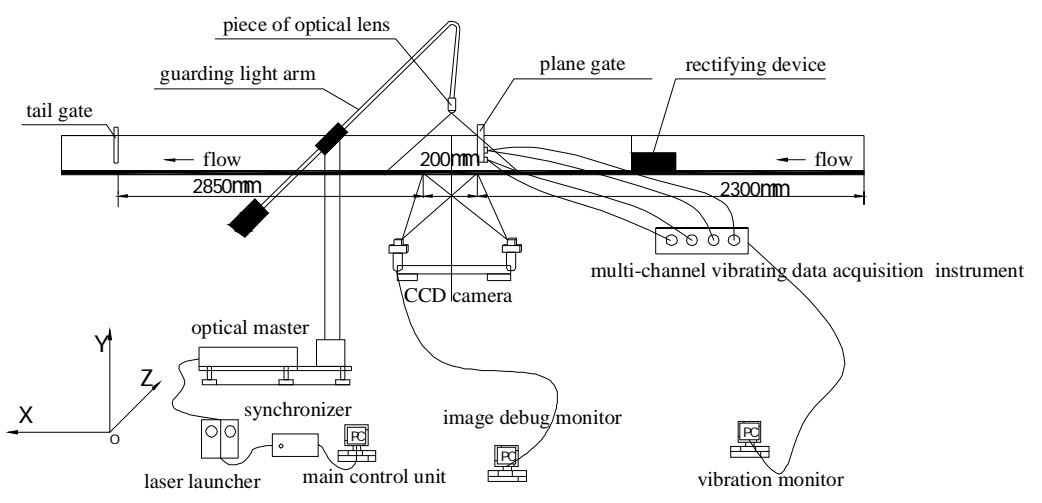

Fig.2. General arrangement of experiment

After the flow is stable, the test starts recording data at the same zero time. Combining the above two sets of measuring systems, the distribution of the flow velocity field and the gate vibration parameters are monitored simultaneously. The general arrangement of experiment is shown in Fig. 2. 
Five experiment conditions Run1 to Run5 are set up in the experiment. Every experiment parameters from Run1 to Run5 are shown in Table 1 in which $\mathrm{e}_{1}$ and $\mathrm{e}_{2}$ are the opening of measured gate and tail gate respectively in the flume.

Table 1 Experiment parameters

\begin{tabular}{cccc}
\hline $\begin{array}{c}\text { Experiment } \\
\text { condition }\end{array}$ & $\begin{array}{c}\text { Discharge } \\
\left(10^{6} \mathrm{~mm}^{3} / \mathrm{s}\right)\end{array}$ & $\begin{array}{c}e_{1} \\
(\mathrm{~mm})\end{array}$ & $\begin{array}{c}e_{2} \\
(\mathrm{~mm})\end{array}$ \\
\hline Run1 & 3.40 & 30 & 40 \\
Run2 & 2.16 & 15 & 25 \\
Run3 & 2.16 & 30 & 25 \\
Run4 & 2.16 & 45 & 25 \\
Run5 & 1.51 & 30 & 15 \\
\hline
\end{tabular}

\section{Flow field}

The 3D-PIV technique is used to measure flow field. Two kinds of more comparable Run2 and Run3 (slotted) are analyzed with the laser frequency $0.97 \mathrm{HZ}$. In the velocity vector diagram behind the gate, $x=0 \mathrm{~mm}$ is the axis location of gate. To reduce the effect of the water fluctuation on PIV testing, the top surface of the flume is covered with a piece of plexiglass, which also has great benefits for laser positioning. The velocity vector diagrams for slotted Run2 and Run3 are shown in Fig. 3.

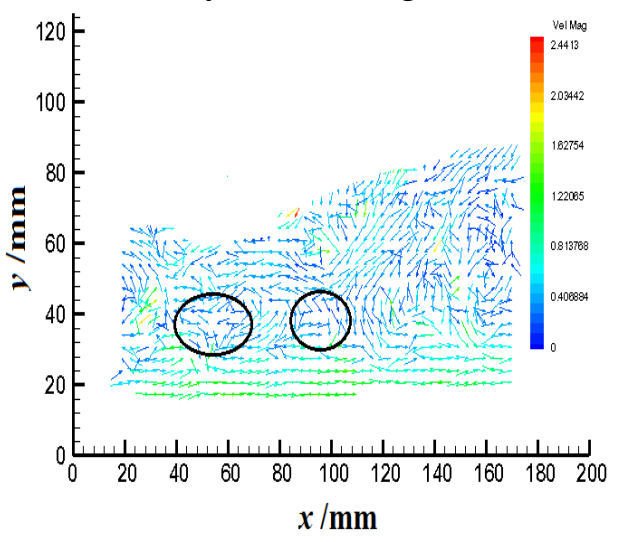

(a) Run2 ( $z=0 \mathrm{~mm}$ section)

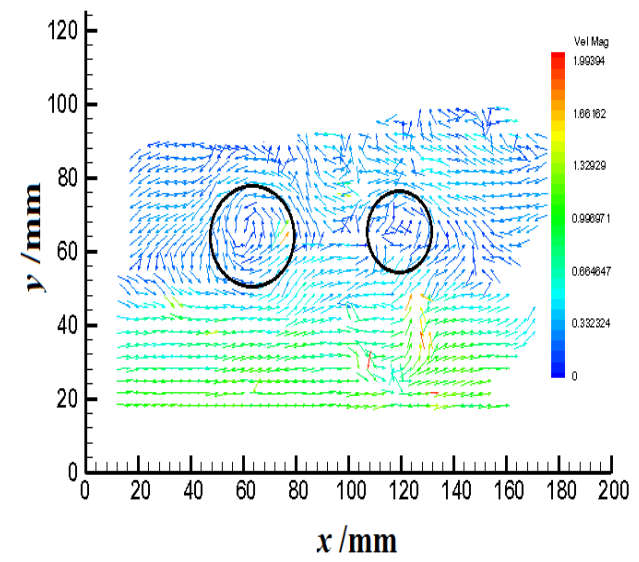

(c) Run3 ( $z=0 \mathrm{~mm}$ section)

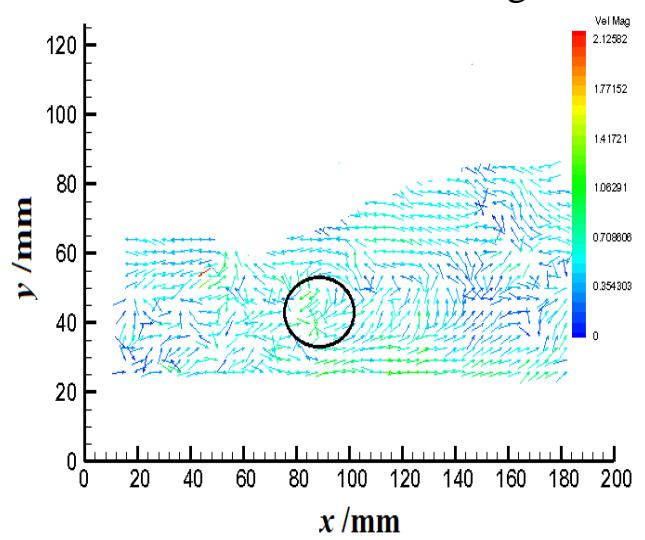

(b) Run2 ( $z=35 \mathrm{~mm}$ section)

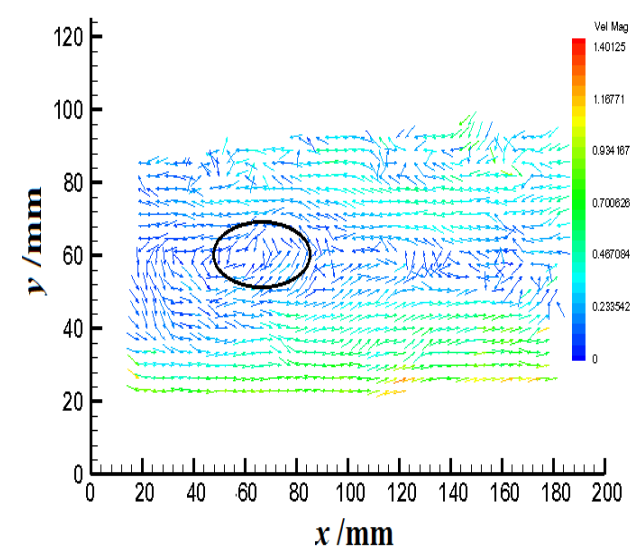

(d) Run3 ( $z=35 \mathrm{~mm}$ section)

Fig.3. Velocity vector diagram behind gate at 20.6 s(slotted)

The average velocity in Run2 with submerged jump behind gate is the largest in the five runs. The surface aeration of flow behind the gate is strong, and has the larger eddies. The free surface flow of Run3 is relatively flat, so Run2 and Run3 are selected to be compared. After removing the pseudo vector and cross-correlation treatment, Fig.3 (a) and (c) show respectively the velocity vector diagrams for the two Runs with slot. The velocity magnitude and direction of tracer particle, the distribution of the vortex, and the water surface profile can be obtained from velocity vector diagram in 
$200 \mathrm{~mm} \times 200 \mathrm{~mm}$ range behind the gate. Due to the different Runs, the center height of vortex near the gate is also different. The vortex center height in Run3 is higher than Run2, which shows a close relationship with the plane gate opening. Under the same condition of the discharge and the opening of the tail gate, the smaller the plane gate opening is, the lower the vortex center height is.

Fig.3 (b) and (d) are the velocity vector diagrams at the section $z=35 \mathrm{~mm}$ for the slotted Run2 and Run3 respectively at 20.6s. Comparing with the velocity vector at the section $z=0 \mathrm{~mm}$, the former's water surface fluctuation is more gentle, and the maximum velocity in $x$-direction is reduced, which results in the decreasing of the vortex energy at the section $z=35 \mathrm{~mm}$. Vortex near the gate in the internal flow field at the section $z=35 \mathrm{~mm}$ is farther away $y$-axis than that at the section $z=0 \mathrm{~mm}$. Accordingly, the following vibration displacement changes can be inferred. The vibration displacement amplitude at point $\mathrm{C}$ is smaller than that at point $\mathrm{A}$, while the amplitude at point $\mathrm{D}$ is smaller than at point $\mathrm{B}$, which is consistent with the subsequently measured result of the gate vibration displacement.

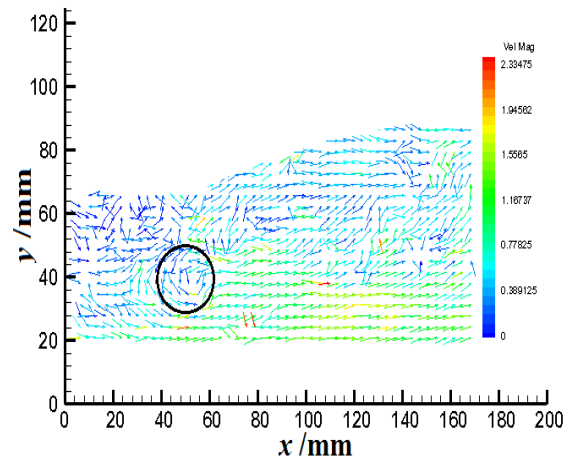

Fig.4. Velocity vector diagram at section $z=0 \mathrm{~mm}$ for Run2 at $20.6 \mathrm{~s}$ (unslotted)

Fig. 4 shows the velocity vector diagram at section $z=0 \mathrm{~mm}$ for unslotted Run2 at 20.6s. Comparing the velocity vector diagrams of Fig.3 (a) with slot and Fig.4 without slot, it can be seen that the position of the vortex has no obvious change, but only one vortex information can be captured at section $z=0 \mathrm{~mm}$ in Fig. 4 without slot. So the effect of gate slot on the range of the velocity vector is mainly just below the gate.

\section{Gate vibration}

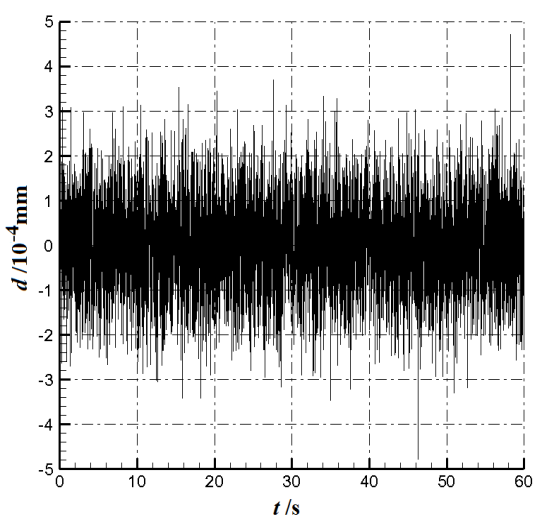

(a) point $\mathrm{A}$

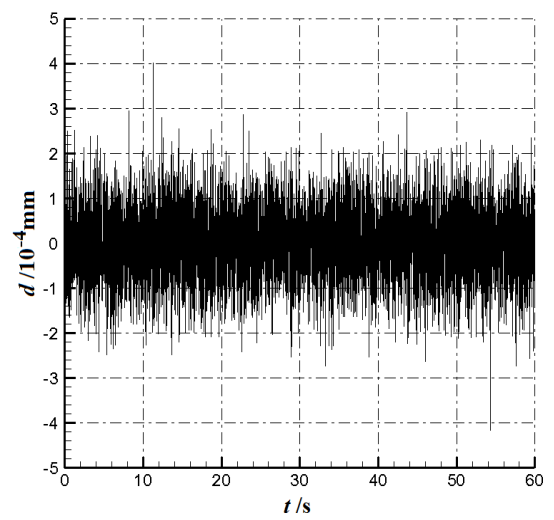

(b) point B

Fig.5. Vibration displacement process in Run2(slotted)

Fig.5 shows the time history of vibration displacement of gate with slot in Run2. It can be seen from Fig.5 that the amplitude of vibration displacement at point $\mathrm{A}$ is larger than that at point $\mathrm{B}$. In addition, the moment to reach the vibration peak value for point $\mathrm{A}$ and $\mathrm{B}$ is not the same, because the dynamic responses at different monitoring locations of the gate acted by the vortex reaches the maximum value at difference time. 


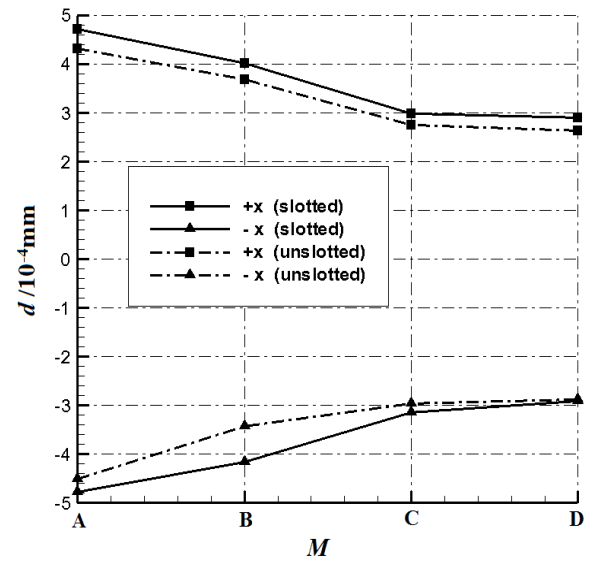

(a) Four points on the gate for Run2

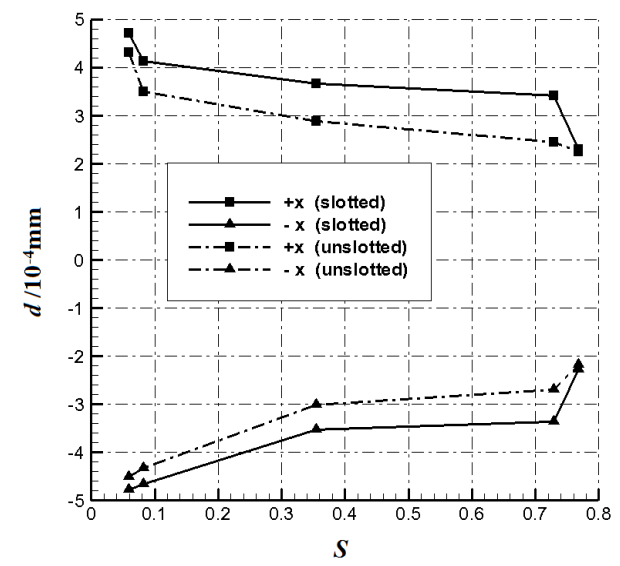

(b) Point $\mathrm{A}$ for different underflow ratio $\mathrm{S}$

Fig.6. Amplitude of vibration displacement

Fig.6 shows the amplitude of vibration displacement of gate. In Fig.6 (b), underflow ratio $S$ is used to access to the characteristics of gate vibration because of submerged flow. Comprehensively considering the upstream and the downstream water head, define underflow ratio $S$ as

$$
S=\frac{h_{t}-h_{c}^{\prime \prime}}{H_{0}-h_{c}^{\prime \prime}}
$$

where, $h_{t}$ is the downstream water depth, $h_{c}$ "the conjugate water depth of the contraction section and $H_{0}$ the total head.

Fig.6 (a) shows the extreme value of vibration displacement at different measured point on the plane gate in Run2. It shows that the extreme value of measured point vibration on $z=0 \mathrm{~mm}$ line is larger than that on $z=35 \mathrm{~mm}$ line for the same $y$-coordinate. That is, the value at point $\mathrm{A}$ is larger than that at point $\mathrm{C}$, and the value at point $\mathrm{B}$ is larger than that at point $\mathrm{D}$. If the $z$-coordinate is the same, the extreme value of measured point vibration on $y=10 \mathrm{~mm}$ line is larger than that on $y=60 \mathrm{~mm}$ line. That is, the value at point $A$ is larger than that at point $B$, and the value at point $C$ is larger than that at point $D$. These are determined by the distance from the vortex center to the measuring point on the gate. The $y$-coordinate center of the vortex behind the gate is about $40 \mathrm{~mm}$ at $z=0 \mathrm{~mm}$ section, while distance of point $\mathrm{A}$ in $y$-direction from the vortex center is $15 \mathrm{~mm}$, and point $\mathrm{B}$ is $35 \mathrm{~mm}$. Therefore, the closer the measured point on the gate to the vortex center is, the larger the extreme value of gate vibration displacement is. In the same Runs, the extreme value of gate vibration displacement without slot is less than that with slot to some extent.

Fig.6 (b) shows the amplitude of vibration displacement at point A for different underflow ratio $S$. It can be seen that the extreme value of vibration displacement at point A decreases with the increasing of underflow ratio, and the amplitude of vibration displacement without slot is less than that with slot. The variation trends at other three points are the same.

Fig.7 shows the spectrum curve of gate vibration at measured points A and B. After Fast Fourier Transform, the dominant frequencies of vibration displacements at point $\mathrm{A}$, point $\mathrm{B}$, point $\mathrm{C}$ and point D are 185.5 HZ, 283.2 HZ, 185.5 HZ and 224.6HZ respectively in Run2 with slot. The dominant frequencies of vibration displacements of all four points are $234.4 \mathrm{HZ}$ in the Run2 without slot. In Run 3 with slot, the dominant frequencies of vibration displacements at point $\mathrm{A}$, point $\mathrm{B}$, point $\mathrm{C}$ and point $\mathrm{D}$ are 205.1HZ、380.9HZ、283.2HZ、517.6HZ respectively. In unslotted condition, the dominant frequencies of vibration displacements at all four points are $273.4 \mathrm{HZ}$. The vibration frequencies have the similar characteristics in Run2 and Run3. In slotted condition, the dominant frequency of vibration displacement of plane gate is related with the location of measured point, and the closer to the edge of 
gate, the smaller the frequency. In unslotted condition, the dominant frequencies at four measuring points trend to accord.

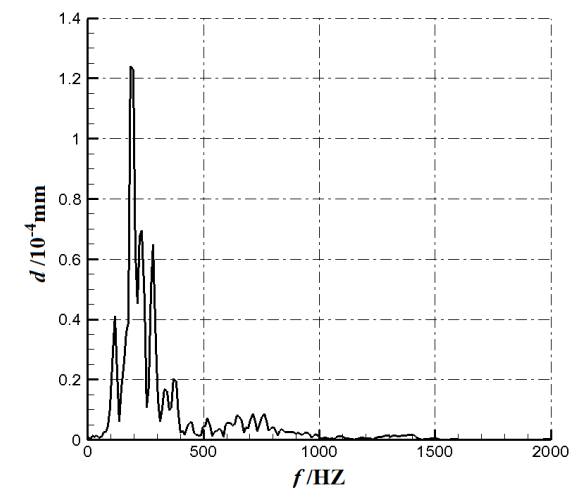

(a) Point A

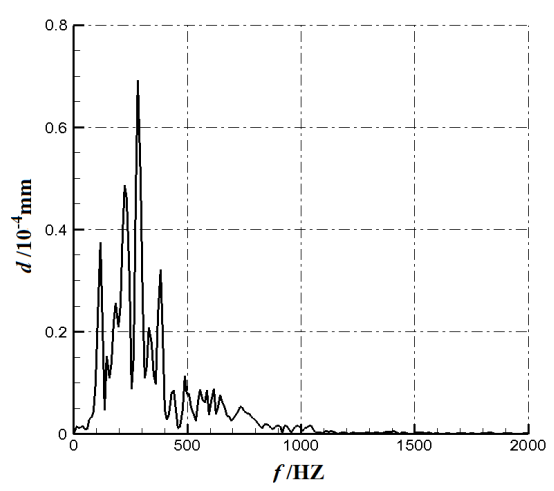

(b) Point B

Fig.7. Spectrum curve of gate vibration at measuring points

Root Mean Square $(R M S)$ and Standard Deviation $(\sigma)$ are used to assess the effective value and discrete degree of vibration displacement $d$. The two statistical parameters are defined as

$$
R M S=\sqrt{\frac{1}{N} \sum_{i=1}^{N} d_{i}^{2}}, \sigma=\sqrt{\frac{1}{N} \sum_{i=1}^{N}\left(d_{i}-\bar{d}\right)^{2}}
$$

For slotted or unslotted condition, the two statistical parameters in Run2 are shown in Table 2. The magnitude trend of the statistical parameters are consistent with the rule of peak value. The vibration displacement is positive or negative relative to the equilibrium position in the $x$-direction, consequently, the $R M S$ and $\sigma$ are the same basically.

Table 2 The statistic parameters

\begin{tabular}{cccc}
\hline \multirow{2}{*}{ Conditions } & \multirow{2}{*}{$\begin{array}{c}\text { Statistical } \\
\text { Parameters }\end{array}$} & point A & point B \\
& $R M S(\mathrm{~mm})$ & $1.06 \mathrm{E}-04$ & $8.40 \mathrm{E}-05$ \\
\multirow{2}{*}{ Slotted } & $\sigma(\mathrm{mm})$ & $1.06 \mathrm{E}-04$ & $8.40 \mathrm{E}-05$ \\
& $R M S(\mathrm{~mm})$ & $9.98 \mathrm{E}-05$ & $6.96 \mathrm{E}-05$ \\
\multirow{2}{*}{ Unslotted } & $\sigma(\mathrm{mm})$ & $9.98 \mathrm{E}-05$ & $6.96 \mathrm{E}-05$ \\
\hline
\end{tabular}

\section{Conclusions}

(1) According to the experimental results by 3D-PIV technique and the multi-channel vibrating data acquisition system, the vibration displacement amplitude of the plane gate mainly depends on the central position and the energy of vortex in the downstream of the gate.

(2) For all the vibrating test points on the gate, the moments to reach the peak value are not the same. The dynamic responses at different monitoring locations of the gate acted by the vortex reaches the peak value at difference time.

(3) In slotted condition, the dominant frequency of vibration displacement of plane gate is associated with the location of measuring point. The closer to the edge of gate is, the smaller the frequency is. In unslotted condition, the dominant frequencies at four measured points trend to accord.

(4) The extreme value of vibration displacement at measured point decreases with the increasing of underflow ratio. The existence of gate slot aggravates the vibration of the gate. In the hydraulic engineering, the size of the gate slot needs to be reasonably designed in order to avoid the adverse flow pattern in the gate slot to lead to the instability of the structure. 


\section{Acknowledgement}

Financial support provided by the National Natural Science Foundation (51369013) is gratefully acknowledged.

\section{References}

[1] Thang Nguyen D. Gate Vibrations due to Unstable Flow Separation [J]. Journal of Hydraulic Engineering, 116(1990): 342-361

[2] Billeter P., Staubli T. Flow-induced multiple-mode vibrations of gates with submerged discharge [J]. Journal of Fluids and Structures, 14 (2000): 323-338

[3] Kostecki S W. Numerical analysis of hydrodynamic forces due to flow instability at lift gate [J]. Archives of Civil and Mechanical Engineering, XI (4) (2011): 943-961.

[4] Ozalp C, Pinarbasi A, Sahin B. Experimental measurement of flow past cavities of different shapes[J].Experimental Thermal and Fluid Science, 34 (2010): 505-515.

[5] Yataghene Mourad, Francine Fayolle, Jack Legrand. Flow patterns analysis using experimental PIV technique inside scraped surface heat exchanger in continuous flow condition [J].Applied Thermal Engineering 31(2011):2855-2868.

[6] Baum E, Peterson B, Surmann C, et al. Investigation of the 3D flow field in an IC engine using tomographic PIV [J].Proceedings of the Combustion Institute 34(2013):2903-2910

[7] Tang Yu-feng, Tian Mao-cheng, Zhang Guan-min. PIV experimental research of flow structure in rectangular channel with transversely placed spiral coil insert [J]. Journal of Hydrodynamics, 24(4)(2012):518-525.

[8] Druault Philippe, Germain Gregory, Facq Jean-Valery. PIV measurements combined with the motion tracking technique to analyze flow around a moving porous structure[J]. Journal of Fluids and Structures 56(2015):190-204

[9] Ayati A A, kolaas J, Jensen A, Johnson G.W. A PIV investigation of stratified gas-liquid flow in a horizontal pipe[J].International Journal of Multiphase Flow 61(2014):129-143

[10] Watanabe R., Gono T, Yamagata T, Fujisawa N. Three-dimensional flow structure in highly buoyant jet by scanning stereo PIV combined with POD analysis. International [J].Journal of Heat and Fluid Flow 52(2015):98-110

[11]Thien Nguyen D, Souad Harmand. PIV measurements in a turbulent wall jet over a backward-facing step in a three-dimensional, non-confined channel [J].Flow Measurement and Instrumentation 42(2015):26-39

[12] Ferguson Tom Mitchell, MacFarlane Gregor, Fleming Alan, Penesis Irene. PIV investigation of 3-dimensional flow within an oscillating water column [J]. International of Marine Energy, 11(2015):120-131

[13] Zhang Jinfeng, Chang Lu, Jerome MAA Peng-Yea. Experimental study on lid-driven cavity flow at high Reynolds number [J]. Advances in Water Science, 26(2) (2015):250-256. 